\title{
Antibacterial activity of manuka honey on the growth of pure and mixed bacterial biofilms formed by sulphate reducing bacteria and Escherichia coli
}

\author{
S. Alkhalil, M. Chopra and V. Zinkevich \\ IBBS, School of Pharmacy and Biomedical Sciences, University of Portsmouth, Portsmouth PO12DT
}

There is increasing evidence that bacteria inhabiting the mucosal surfaces of the human colon play a role in the development of inflammatory bowel disease (IBD). The ability of these bacteria to form biofilms increases their chance of survival at high concentrations of antibiotics. ${ }^{(1,2)}$ Sulphate reducing bacteria (SRB) have been observed to be present in high level in IBD patient indicating the possible role of these bacteria in the colon infection. ${ }^{(3)}$ The induction of inflammation by adherence Escherichia coli (E. coli) to intestinal epithelial cells also has been reported. ${ }^{(4)}$ Manuka honey is well known for its antibacterial properties and the effect is attributed to a joint action of methylglyoxal (MGO) and other unknown compounds, which provide extra antiseptic and therapeutic properties against several antibiotic resistance microorganisms.

The present study was conducted to examine the effect of manuka honey UMF® $5+$ and manuka honey UMF® $15+$ on the growth of planktonic and biofilm cultures of SRB strain Desulfobrio indonensis and E. coli. Both pure and mixed cultures were studied. Response was examined at varying dose of honey to determine the Minimum Inhibitory Concentration (MIC). Manuka honey $\mathrm{UMF}{ }^{\circledR} 15+$ showed the maximum antimicrobial activity at final concentration of $11 \% \mathrm{w} / \mathrm{v}$ in pure $D$. indonensis planktonic and biofilm culture. However, the microbial growth of pure planktonic and biofilm E. coli culture as well as the mixed $D$. indonensis and E.coli was maximally inhibited at final concentration of $16 \% \mathrm{w} / \mathrm{v}$ honey with no antibacterial activity of honey observed at low concentration of $5 \% \mathrm{w} / \mathrm{v}$. In contrast, Manuka UMF® $5+$ showed approximately no effect on pure and mixed planktonic and biofilms $E$. coli culture at $25 \%$ w/v compared to its effect on the growth of pure D. indonensis at $20 \%$ w/v and mixed at $25 \%$ $\mathrm{w} / \mathrm{v}$ in both planktonic and biofilms.

In conclusion, present investigation has highlighted a protective role of manuka honey against mixed bacterial biofilms. The antimicrobial activity of manuka honey was observed in both planktonic and biofilms bacterial culture and the effect was dependent on the concentration and UMF number of the honey.

1. Swidsinski AJ (2005) Clin. Microbiol. 43(7), 3380-3389.

2. Dongari-Bagtzoglou A (2008) Expert Rev Anti Infect Ther. 6(2), 201-208.

3. Roediger WEW. et al. (1993) Gastroenterology 104(3), 802-809.

4. Rolhion N \& Darfeuille-Michaud A (2007). Inflamm Bowel Dis 13(10), 1277-1283. 